\title{
Thermostructural Analysis of the Muriaé Hydrographic Basin
}

Hellen Rosa Barboza ${ }^{1}$, Suze Nei Pereira Guimarães ${ }^{2}$, Fabio Pinto Vieira ${ }^{2}$

1 Universidade Federal Fluminense - Instituto de Geociências

${ }^{2}$ Observatório Nacional - ON/MCTIC - Departamento de Geofísica - Laboratório de Geotermia (LabGeot)

Copyright 2019, SBGf - Sociedade Brasileira de Geofísica

This paper was prepared for presentation during the $16^{\text {th }}$ International Congress of the Brazilian Geophysical Society held in Rio de Janeiro, Brazil, 19-22 August 2019.

Contents of this paper were reviewed by the Technical Committee of the $16^{\text {th }}$ International Congress of the Brazilian Geophysical Society and do not necessarily represent any position of the SBGf, its officers or members. Electronic reproduction or storage of any part of this paper for commercial purposes without the written consent of the Brazilian Geophysical Society is prohibited.

Abstract

The present work deals a thermostructural analysis of the fluminense part of the Muriae hydrographic basin. Geothermal and magnetic studies were applied include crust thermal analysis with investigations from geothermal gradients and heat flow. Geophysical interpretations techniques in residual magnetic field as vertical derivative, analytic signal, Euler deconvolution and spectral analysis involved the magnetic analysis. The results obtained in this study, reveals two distinct thermostructural zones. A first zone identified are be localized in the midwest part of study area, that is compose with the part of Atlantic Plateau (W) and part of depression interplanar zone (center). This zone are marked by NE-SW fracturing with N-S tendency, the values mean to heat flow is a $60 \mathrm{~mW} / \mathrm{m}^{2}$, thermal conductivity is a $3 \mathrm{~W} / \mathrm{mK}$ and the $3-8 \mathrm{~km}$ to depth of magnetic sources (deepest sources of study area). The second distinct thermostructural zone localize in SE region of study area. Is compose by sedimentary sequences and the Campos basin boundary. These structural lineaments NE-SW have a E-W tendency, the values mean to heat flow is a $80 \mathrm{~mW} / \mathrm{m}^{2}$, thermal conductivity is a $2,5 \mathrm{~W} / \mathrm{mK}$ and the $4-5 \mathrm{~km}$ to depth of magnetic sources.

This systematic difference of the magnetic sources depth between these two regions substantially evidenced structurally, points to the performance of mechanisms related to the crustal thermal regime. Assessment of geothermal gradient variation due to climate change showed a variation of $2.9^{\circ} \mathrm{C}$ in the last 55 years for the region.

\section{Introdução}

As bacias hidrográficas, como um sistema individualizado, podem ser consideradas como fontes de dados relevantes para obtenção de informações sobre evolução da crosta terrestre. Uma vez que as formas de relevo, que definem os canais hidrográficos, são oriundas das interações entre os processos tectônicos, pedogênicos, intempéricos e termal daquela região.

Utilizando parâmetros físicos de calor e magnetismo é possível explicar o contexto termomagnético das rochas em subsuperfície, agregando às características hidrogeológicas informações muitas vezes não evidenciadas em estudos geológicos de superfície.
O rio Muriaé é o último afluente do rio Paraíba do Sul antes de sua foz no Oceano Atlântico. A bacia hidrográfica do rio Muriaé, está localizada nos estados de Minas Gerais e Rio de Janeiro. Possui uma área aproximadamente de $8.200 \mathrm{~km}^{2}$. Este estudo foi realizado na porção Centro-Sul da bacia hidrográfica do Rio Muriaé, localizada na região noroeste do estado do Rio de Janeiro.

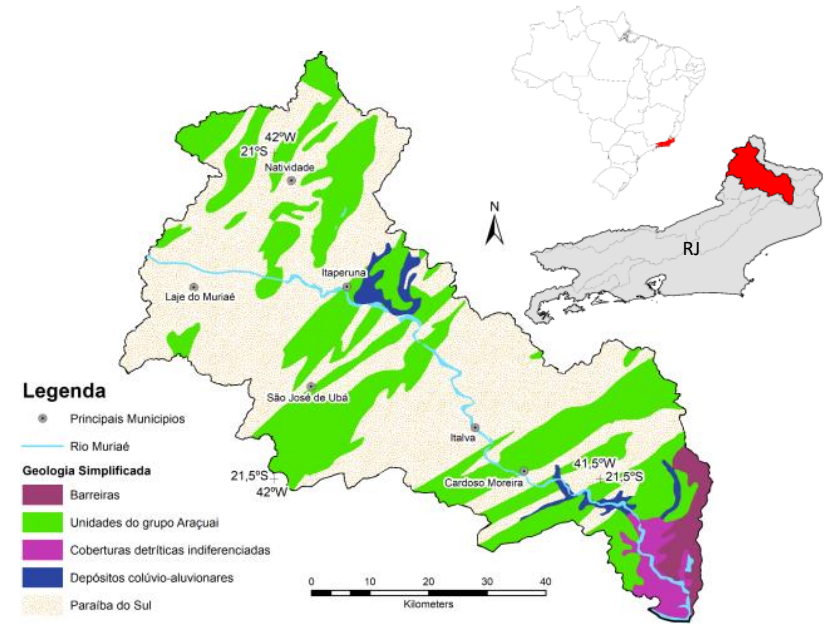

Figura 1 - Localização geográfica e Geologia simplificada da região fluminense da bacia hidrográfica do rio Muriaé (adaptado de CPRM, 2014)

A região central da bacia é marcada por uma região da depressão interplanáltica dos rios Pomba-Muriaé (Brenner et al., 1980, Ross, 1985) e o arcabouço tectônico é marcado pelos grandes lineamentos regional com direção E-NE até N-NE (zonas de cisalhamento) correspondendo a extensas e espessas faixas de rochas cataclásticas. Geologicamente é marcado pelas unidades neoproterozóicas Araçuaí e Paraíba do Sul.

O presente trabalho utilizou dados geotérmicos e aeromagnéticos, como objetivo de obter informações sobre a evolução termoestrutural da bacia hidrográfica do rio Muriaé.

\section{Métodos Geofísicos Empregados ao Estudo}

Método Geotérmico: As informações geotérmicas fornecem importantes dados sobre anomalias relacionadas ao regime térmico da crosta e a fenômenos geológicos e tectônicos nelas associados.

Base de Dados Geotérmicos: a base de dados para caracterização do regime geotérmico da crosta na região de estudo consta de vinte e dois (22) poços disponibilizados pelo Sistema de Informações de Águas Subterrâneas (SIAGAS) do Serviço Geológico do brasil (CPRM) e oito (8) poços disponibilizados pelo Laboratório 
de Geotermia do Observatório Nacional (LabGeot), localizados no de estudo contexto (Figura 2).

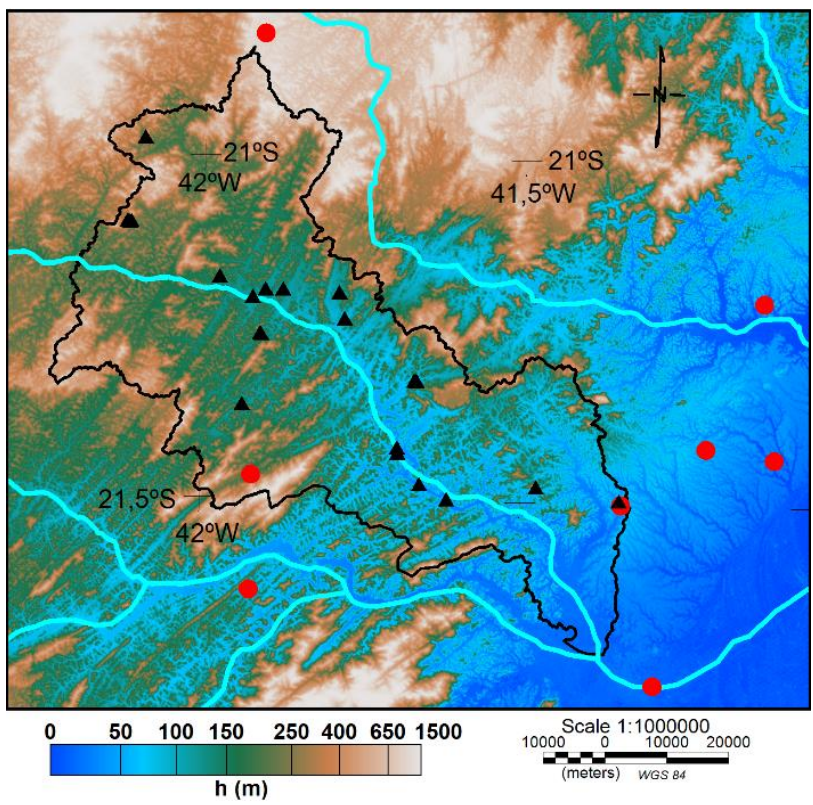

Figura 2 - Modelo Digital do Terreno com a distribuição espacial dos dados geotérmicos utilizados no estudo (Valeriano, 2008). Os triângulos pretos referem-se aos dados disponibilizados pelo SIAGAS e os círculos vermelhos referem-se aos dados do LabGeot/ ON.

Gradiente Geotérmico: o cálculo do gradiente geotérmico consiste na taxa de variação da temperatura com a profundidade (medida em poço).

$$
\Gamma=\frac{T_{z}+T_{0}}{z}
$$

Em que $T_{z}$ é a temperatura na profundidade $z ; T_{0}$ é a temperatura na superfície e $z$ a profundidade do poço.

Condutividade Térmica: Os valores de condutividade térmica foram obtidos junto ao departamento de geologia da Universidade Estadual do Rio de Janeiro, que também forneceu a descrição litológica dos poços analisados. A Tabela 1 apresenta os valores da condutividade térmica para as principais litologias da região de estudo. Este valor representativo é calculado por média ponderada das litologias presentes no poço em virtude da não homogeneidade vertical de condutividade.

Fluxo de Calor: o fluxo de calor é a transmissão de energia por unidade de área e de tempo e se quantifica pelo produto do gradiente geotérmico pela condutividade média ponderada de cada poço como mostrado abaixo.

$$
q=\Gamma \cdot \lambda
$$

Onde: $q$ é o Fluxo de Calor em $\mathrm{mW} / \mathrm{m}^{2}$, $\Gamma$ é o Gradiente Geotérmico em ${ }^{\circ} \mathrm{C} / \mathrm{K}$ e $\lambda$ é a Condutividade Térmica em W/mK.
Tabela 1 - Principais tipos litológicos da região de estudo, segundo Gomes (2003) e seus valores de condutividade térmica

\begin{tabular}{|c|c|c|c|}
\hline \multirow{2}{*}{ Grupo } & \multirow{2}{*}{ Tipo Litológico } & \multicolumn{2}{|c|}{ Condutividade (W/(m K)) } \\
\hline & & Média & $\sigma$ \\
\hline \multirow[t]{6}{*}{ Rochas Sedimentares } & Areia Média Grossa & 2,8 & 0,8 \\
\hline & Areia Média Argilosa & 2,2 & 0,8 \\
\hline & Arenito Médio & 2,7 & 0,8 \\
\hline & Não Classificados & 2,7 & 0,8 \\
\hline & Sedimentos Fluviais & 2,7 & 0,8 \\
\hline & Siltito & 2,7 & 1,3 \\
\hline \multirow[t]{3}{*}{ Metamórfica de Baixo Grau } & Anfibolito & 3,3 & 1,6 \\
\hline & Calcissilicática & 2,4 & 1,0 \\
\hline & Quartzito & 3,1 & 1,0 \\
\hline \multirow[t]{8}{*}{ Metamórfica de Alto Grau } & Biotita Gnaisse & 3,5 & 2,0 \\
\hline & Gnaisse Bandado & 3,0 & 2,0 \\
\hline & Gnaisse Facoidal & 4,3 & 2,4 \\
\hline & Gnaisse Granitico & 3,1 & 2,1 \\
\hline & Gnaisse Leucocrático & 2,8 & 1,2 \\
\hline & Gnaisse Mesocrático & 3,9 & 1 \\
\hline & Granada-Gnaisse & 4,4 & 3,0 \\
\hline & Granulito & 3,3 & 1,7 \\
\hline \multirow[t]{5}{*}{ Rochas Igneas Ácidas } & Granito & 3,1 & 1,3 \\
\hline & Granito diferenciado & 2,7 & 1.5 \\
\hline & Granito Porfiritico & 2,6 & 0,7 \\
\hline & Granitóide & 3,0 & 1,7 \\
\hline & Granitóide Tonalitico & 3,1 & 1,3 \\
\hline Rochas İgneas Básicas & Diabásio & 2,6 & 0,7 \\
\hline
\end{tabular}
média correspondentes.

Método Magnetométrico: Este método mede a intensidade do campo magnético terrestre, que sofre influência das rochas em profundidade, aumentando ou diminuindo o seu valor, conforme a composição destas rochas e os contrastes de susceptibilidade magnética existente entre as mesmas. Para este estudo, foram utilizados dados área de dois aerolevantamentos: Espírito Santo (código 1093, realizado em 2010) e Rio de Janeiro (código 1117, realizado em 2012).

As características de ambos aerolevantamentos são: direção de linhas de voo N-S, espaçamento de 500m e altura nominal de voo de $100 \mathrm{~m}$. As linhas de controle foram adquiridas perpendicularmente a aquisição (E-W) com espaçamento de $10 \mathrm{~km}$. O software utilizado para processamento e aplicação de técnicas geofísicas e filtragens foi o Oasis Montaj da Geosoft e a junção dos grids de medida foi realizada com a técnica de "suture".

Os dados digitais dos projetos utilizados que foram cedidos pela CPRM ao LabGeot, para fins de estudo e pesquisa, já foram adquiridos com tratamento inicial e correções dos erros de posicionamento, nivelamento das linhas, deriva de voo, remoção do IGRF e micronivelamento.

Processamento A partir da malha micronivelada do campo magnético anômalo foram gerados grids de dados interpolados pelo método de Mínima Curvatura com tamanho de célula de $125 \mathrm{~m}$. Foram aplicados técnicas interpretativas de derivada vertical e amplitude do sinal analítico ao campo magnético anômalo.

Tais atributos foram manipulados na forma de mapas, objetivando a interpretação de anomalias associadas a falhas e diques, realçadas através destas técnicas. Para verificação das profundidades das fontes anômalas de interesse (lineamentos) foi utilizada a técnica de Deconvolução de Euler e análise espectral.

Derivada Vertical do Campo Magnético Anômalo (Gunn, 1975): a técnica de derivada vertical é o vetor resultante de realce das altas frequências do sinal magnético onde a anomalia magnética $(B)$ é transformada linearmente por 
meio da primeira derivada da componente vertical $(z)$ do campo magnético anômalo, ou seja, é a taxa de variação do campo magnético anômalo a medida que se distancia ou aproxima verticalmente da sua fonte (Blakely, 1996). Assim é possível mostrar que a derivada vertical de ordem $n$ é igual a transformada Fourier do potencial (no caso campo magnético $-B$ ) vezes um fator $\left(\mathrm{k}^{\mathrm{n}}\right)$.

$$
I\left[\frac{\partial^{n} B}{\partial z^{n}}\right]=|k|^{n} I[B]
$$

O mapa da derivada vertical do campo magnético anômalo permite visualizar os contrastes entre os diferentes domínios magnéticos identificados a partir da imagem do campo magnético anômalo.

Amplitude do Sinal Analítico (Thompson, 1982): Esta técnica geofísica é basicamente a raiz da soma do módulo das três componentes das derivadas direcionais (horizontal$x, y$ e vertical-z) do campo magnético anômalo, caracterizando-se como um pacote de energia.

$$
A(x, y, z)=\sqrt{\left|\frac{\partial B}{\partial x}\right|^{2}+\left|\frac{\partial B}{\partial y}\right|^{2}+\left|\frac{\partial B}{\partial z}\right|^{2}}
$$

Esta amplitude $(A)$ tem uma forma sobre corpos causadores de anomalias que depende apenas de suas localizações espaciais e não das direções de magnetização dos mesmos, por isso essa ferramenta é utilizada para estudo da profundidade e posições das suas fontes magnéticas anômalas.

Deconvolução de Euler (Nettelton e Cannon, 1962; Thompson, 1982, Clark, 1997): esta técnica é utilizada para extrair informações sobre a profundidade das fontes magnéticas. Seu resultado independe da direção e inclinação do campo magnético principal e da orientação de magnetização das fontes anômalas, desta forma esta metodologia é relativamente insensível a pequenas distorções do campo.

Neste processamento foi aplicada esta técnica no grid de campo magnético anômalo filtrado (filtro passaalta), de modo a realçar apenas as fontes de contexto tectônico de interesse, ou seja, falhas estruturais ligadas aos lineamentos magnéticos mapeados na derivada vertical. Para o cálculo se adotou o índice estrutural $\eta=1$ (modelo de dique) e erros de no máximo 10\% para o posicionamento e profundidade das fontes.

Análise Espectral das Fontes Anômalas (Bhattacharyya e Leu, 1975, 1977): foram utilizados dois métodos de análise espectral em conjunto, para determinar a profundidade das fontes magnéticas anômalas na região. $\mathrm{O}$ método de inclinação espectral, que procura separar as fontes magnéticas anômalas em camadas e o método Centróide, que procura encontrar a camada magnética mais profunda na crosta. Assim é possível estimar a espessura das camadas magnéticas, em profundidade, realizando um paralelo com a superfície de Curie.

\section{Resultados}

Geotermia: Os perfis geotérmicos selecionados permitiram a determinação direta das temperaturas nas faixas de profundidade selecionadas. A Figura 3 apresenta exemplos de perfis térmicos de poços utilizados no estudo, eles foram selecionados dentre os poços apresentados na Figura 2.

Nesses perfis as sequências de pontos em azul indicam as distribuições verticais de temperaturas medidas. Os pontos em cor vermelha, na superfície, indicam temperaturas médias anuais para cada local do poço e foram obtidas com base em informações disponíveis em pesquisas meteorológicas locais (INMET).
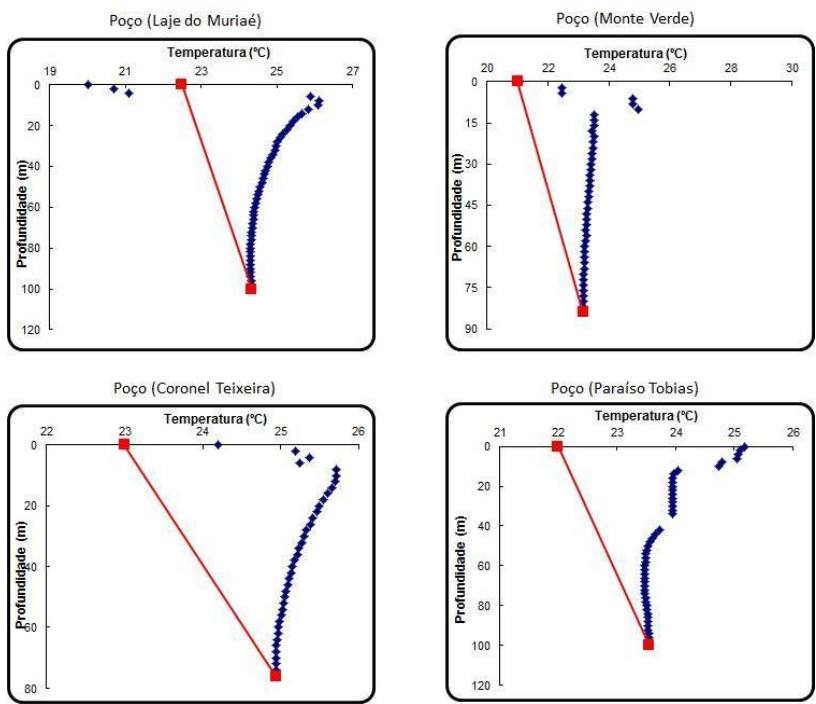

Figura 3 - Exemplos de quatro (4) perfis térmicos de poços utilizados no estudo.

As linhas em cor vermelha, conectam as temperaturas médias anuais da superfície com os respectivos valores observados no fundo do poço. De modo que, a diferença observada entre as temperaturas medidas e o ajuste indicam as perturbações nas temperaturas. Há indícios de que a maior parte das perturbações foram induzidas por transporte de calor associado aos movimentos de água no meio geológico e alterações nas temperaturas induzidas por mudanças climáticas locais.

Análise dos perfis permitiram uma avaliação sobre a variação do gradiente geotérmico devido a mudanças climáticas. De uma maneira geral, modelagens foram realizadas em alguns perfis geotérmicos para estimar essa variação e assim estabelecer relação ao contexto tectônico da área. O melhor ajuste obtido apresentado na Figura 4, foi modelado para o poço localizado na região sul da área de estudo, denominado UENF por se localizar no campus desta universidade. Os resultados mostram uma variação linear da magnitude de $2,9^{\circ} \mathrm{C}$ nos últimos 55 anos.

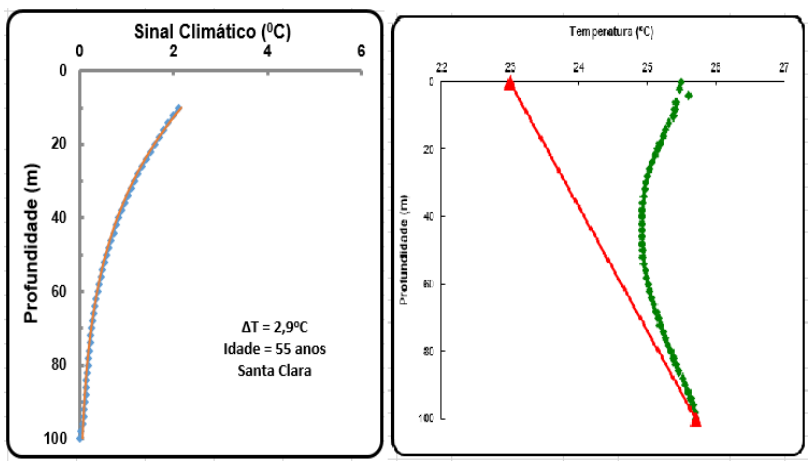

Figura 4 - Gráficos da (a) modelagem térmica de variação do gradiente geotérmico devido a mudanças climáticas (b) distribuição da temperatura em profundidade, para o poço UENF. 
A melhor forma de examinar as variações regionais do gradiente geotérmico e do fluxo de calor é através da elaboração de mapas. A distribuição do gradiente e do fluxo geotérmico em escala regional na região de estudo foi realizada através do método de superfícies numéricas e os resultados são apresentados, respectivamente nas Figuras (5) e (6).

A distribuição do gradiente geotermal da bacia hidrográfica do rio Muriaé indica que valores maiores que $25^{\circ} \mathrm{C} / \mathrm{km}$ ocorrem ao longo da zona de drenagem principal do rio Muriaé, de noroeste a sudeste. A região sul é caracterizada por gradientes térmicos maiores que $30^{\circ} \mathrm{C} / \mathrm{km}$. Feições semelhantes também são observadas na distribuição de fluxo geotérmico, alcançando valores maiores que a média global $\left(>80 \mathrm{~mW} / \mathrm{m}^{2}\right)$ nos locais próximos ao fluxo do rio Muriaé, atingindo seus maiores índices na região SE da bacia.

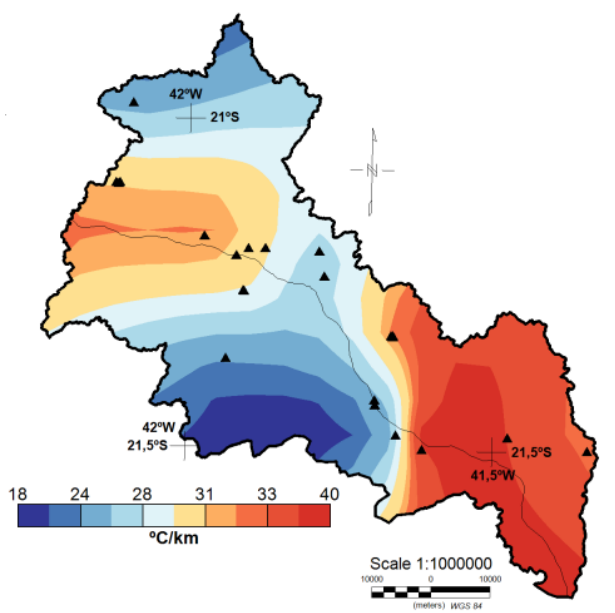

Figura 5 - Mapa do gradiente geotermal da bacia hidrográfica do rio Muriaé (parte fluminense). Os triângulos indicam os poços utilizados no estudo

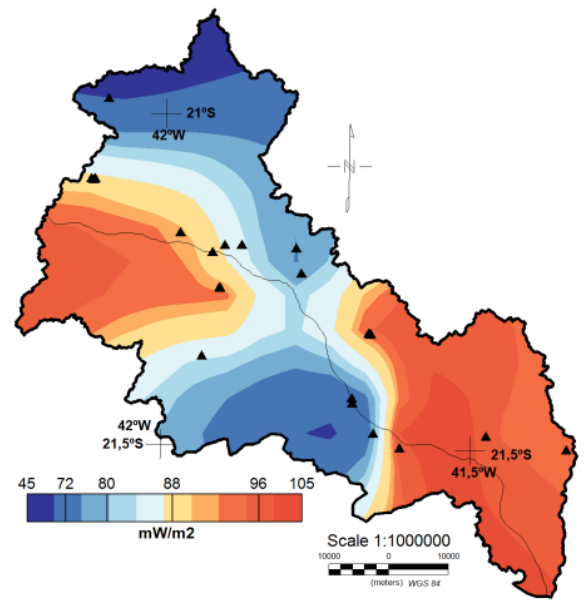

Figura 6 - Mapa do fluxo geotérmico da bacia hidrográfica do rio Muriaé (parte fluminense).

Magnetometria: O mapa do campo magnético residual, apresentado na Figura 7, revela a existência de variações consideráveis na intensidade do campo crustal/litosférico. O Valor de $\pm 150 \mathrm{nT}$ foi obtido como média do campo magnético crustal da bacia hidrográfica do rio Muriaé.

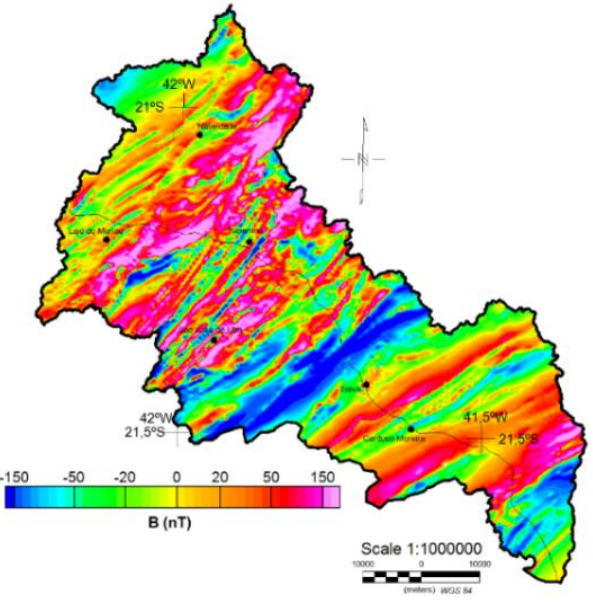

Figura 7 - Mapa do Campo Magnético Anômalo da bacia de Muriaé.

A região é caracterizada por possuir uma zona de altos valores magnéticos na area central da bacia, onde há uma zona de faturamento condensada.

\section{Arcabouço Magnético-Estrutural}

Foi possível observar de forma geral, no mapa de derivada vertical do campo magnético residual, que a direção prioritária dos lineamentos magnéticos é NE-SW (Figura 8). Existe uma diferenciação na angulação desses lineamentos. Na região central da bacia, a angulação dos lineamentos tende para N-S, já na região sudoeste da bacia essa angulação tende para E-W. As principais estruturas tectônicas morfoestruturais estão delimitadas e identificadas na Figura 8 por um traço em amarelo, baseado em Silva 2002.

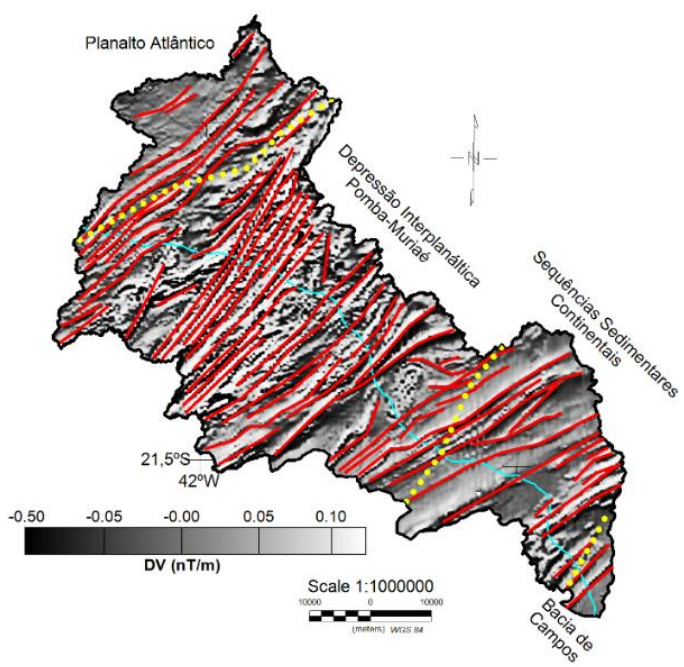

Figura 8 - Mapa da derivada vertical do campo magnético residual da bacia hidrográfica do rio Muriaé (parte fluminense). Os lineamentos magnéticos observados a partir desta técnica geofísica estão realçados na cor vermelha neste mapa. Principais estruturas morfoestruturais (Silva, 2002) estão destacados com linhas pontilhadas na cor amarela.

O modelo digital do terreno (MDT) ilustrado na Figura 2, traz informações em superfície uma vez que observamos que as estruturas topográficas na região 
central da bacia do rio Muriaé chegam a uma altura de 400 m e possui a mesma tendência NE-SW.

A região NW da bacia, é uma área de topografias elevadas, relacionadas com Planalto Atlântico (Silva et al, 2000). Junto a área central da bacia encontramos uma zona de lineamento condensada, com pequenas variações em suas distâncias. O que revela que essa zona sofreu um resfriamento abrupto durante sua formação.

Na região SE da área de estudo podemos verificar que os lineamentos magnéticos sofrem uma pequena angulação para leste coincidindo com o contexto tectônico estrutural de sequências sedimentares até a borda da bacia sedimentar de Campos, onde ocorre o deságue do rio Muriaé no rio Paraíba do Sul.

O mapa da amplitude do sinal analítico, ilustrado na figura 9, também corrobora com o estudo do arcabouço magnético-estrutural da bacia do rio Muriaé, uma vez que podemos aferir na região, um grau de conformidade com as estruturas lineares identificadas no mapa da derivada vertical. Os valores da amplitude do sinal analítico variam de 0.01 a $0.30 \mathrm{nT} / \mathrm{m}$.

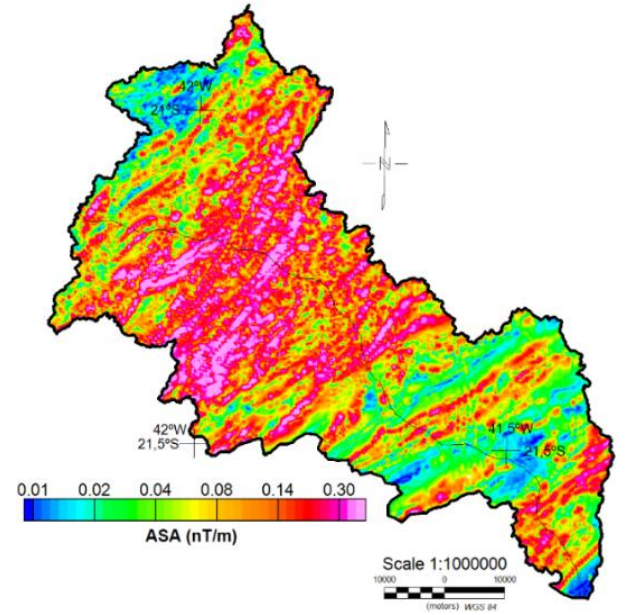

Figura 9 - Mapa da amplitude do sinal analítico do Campo Magnético Anômalo da bacia de Muriaé.

O grau de fraturamento da região também é observado no método geotérmico, onde é possível identificar a movimentação de fluidos subterrâneos em zonas onde não é exatamente o dorso do rio Muriaé. O que comprova esse alto grau de fraturamento da região. Esta observação pode ser determinada a partir da análise de perfis geotérmicos uma vez que o campo termal é afetado pelos efeitos do transporte de calor por advecção provocados por esse movimento em um ambiente favorável (rocha porosas ou fraturadas).

Para um detalhamento das fontes magnéticas de interesse, foi aplicado um filtro passa-alta no grid do campo magnético anômalo, de modo a realçar apenas essas fontes de interesse tectônico-estrutural, ligados a estruturas lineares que por sua vez está em concomitância a lineamentos geoestruturais. Foram utilizadas duas técnicas para calcular a profundidade das fontes magnéticas de modo a verificar os resultados e minimizar os erros.

Para a técnica de Deconvolução de Euler utilizamos o índice estrutural $(n=1)$ que relaciona a modelos de diques e erro máximo de $10 \%$ para o posicionamento e profundidade das fontes. Os resultados são relacionados na parte (a) da figura 10. O cálculo de profundidade das fontes magnéticas por Deconvolução de Euler apresentou valores distribuídos desde a superfície até $4500 \mathrm{~m}$. A maior concentração de estruturas ocorreu no intervalo entre 1000-1300m. Há uma boa correlação entre os lineamentos traçados pela técnica de derivada vertical.

Para a análise espectral foi utilizado janelas móveis do campo magnético crustal para cálculo da profundidade de camadas magnetizadas. Assim para as fontes magnéticas mais profundas, foi delimitado uma profundidade, chamada neste trabalho de profundidade Curie, considerando a principal fonte magnética advinda da magnetita (que possui a temperatura de Curie: $580^{\circ} \mathrm{C}$ ). Os valores para a profundidade de Curie estão ilustrados no mapa da parte (b) da figura 10. Os valores encontrados nessa metodologia estão descritos na tabela 2 .

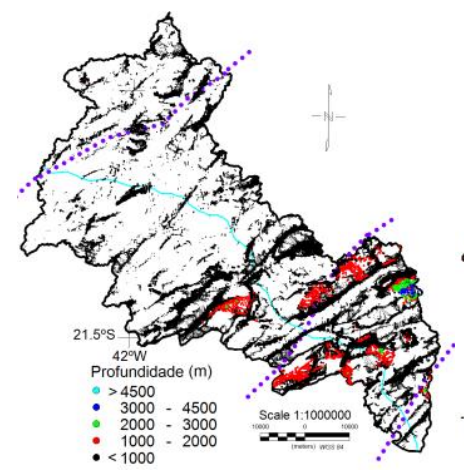

(a)

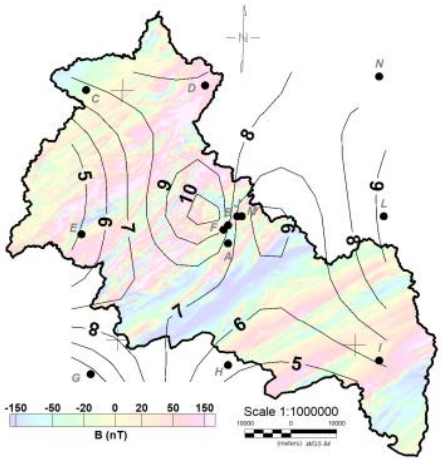

(b)
Figura 10 - Resultados das aplicações de técnicas geofísicas interpretativas (a) profundidades das fontes magnéticas obtidos pelas soluções de Euler aplicado no grid de campo magnético anômalo filtrado (passa-alta) (b) profundidade de Curie obtido por analise espectral do campo magnético anômalo.

Tabela 2: Profundidades para o topo $\left(z_{\text {top }}\right)$ e base $\left(z_{\text {base }}\right)$ para as janelas selecionadas na região de estudo, baseado no método centroide de analise espectral.

\begin{tabular}{|c|c|c|c|c|c|c|c|c|c|}
\hline \multirow{2}{*}{ ID } & \multirow{2}{*}{ Long (W) } & \multirow{2}{*}{ Lat (S) } & \multirow{2}{*}{ Janela $(\mathrm{m})$} & \multicolumn{2}{|c|}{ RASA } & \multicolumn{2}{|c|}{ INTERMEDIARIA } & \multicolumn{2}{|c|}{ PROFUNDAS } \\
\hline & & & & ztop & zbot & ztop & zbot & ztop & zbot \\
\hline A & 41.77 & 21.30 & 80 & * & * & * & * & 3.72 & 9.95 \\
\hline B & 41.77 & 21.27 & 90 & 0.54 & 1.29 & 1.18 & 3.18 & 3.37 & 10.86 \\
\hline C & 42.08 & 21.00 & 20 & 0.25 & 1.23 & 0.38 & 1.57 & 4.21 & 7.71 \\
\hline D & 41.83 & 20.99 & 20 & 0.45 & & 0.82 & 1.69 & 2.60 & 8.82 \\
\hline$E$ & 42.08 & 21.29 & 30 & 0.67 & 1.34 & 0.97 & 1.97 & 2.42 & 4.78 \\
\hline $\mathrm{F}$ & 41.78 & 21.28 & 30 & 0.40 & 1.24 & 0.93 & 1.82 & 2.91 & 6.51 \\
\hline $\mathrm{G}$ & 42.05 & 21.57 & 20 & 0.30 & 0.91 & 1.03 & 2.21 & 6.26 & 10.68 \\
\hline $\mathrm{H}$ & 41.77 & 21.55 & 30 & 0.05 & 0.53 & 0.54 & 2.35 & 3.26 & 5.62 \\
\hline 1 & 41.45 & 21.53 & 30 & 0.65 & 1.50 & 0.86 & 2.17 & 2.18 & 6.22 \\
\hline 1 & 41.75 & 21.25 & 30 & 0.22 & 1.17 & 1.10 & 2.31 & 3.42 & 3.66 \\
\hline$L$ & 41.45 & 21.24 & 30 & 0.66 & 1.28 & 0.91 & 2.00 & 4.14 & 9.39 \\
\hline $\mathrm{M}$ & 41.74 & 21.25 & 80 & 0.59 & 1.69 & 1.82 & 2.93 & 2.42 & 9.04 \\
\hline $\mathrm{N}$ & 41.46 & 20.96 & 30 & 1.67 & 2.40 & 2.77 & 3.80 & 2.25 & 7.49 \\
\hline
\end{tabular}

As fontes magnéticas mais profundas foram encontradas na região central da bacia, onde a camada mais profunda foi delimitada na faixa de 3 a $8 \mathrm{~km}$ aproximadamente. Essa camada está ligada a zona de fraturamento da região. Na região SW da área de estudo, verificamos a existência de fontes magnéticas pontuais onde os valores da profundidade para a camada mais profunda nessa região são da ordem de $4 \mathrm{~km}$, no entanto a base dessa camada não ultrapassa $5 \mathrm{~km}$. Essa característica advém do contexto sedimentar dessa região.

As diferenças sistemáticas de profundidades das fontes magnéticas entre essas duas regiões apontam para atuação de mecanismos relacionados com o regime termal da crosta. 


\section{Considerações Finais}

As diferenças sistemáticas nas profundidades dos corpos magnéticos inferidos e a correlação espacial entre os lineamentos magnéticos regionais e as estruturas topográficas, evidenciam duas zonas termoestruturais distintas: Uma na região centro-oeste da bacia, que é marcada por uma depressão interplanaltica e possui suas fontes estruturais com uma profundidade variando de 3-8 $\mathrm{km}$ apresentando valores de fluxo geotérmico relativamente baixos $\left(60 \mathrm{~mW} / \mathrm{m}^{2}\right)$. Os lineamentos magnéticos identificados NE-SW possuem uma tendência a N-S, além do alto grau de lineamentos magnéticos com pequenas distancias entre si. Essas características são evidências de uma zona geotermicamente estável, cujo resfriamento em sua formação se deu de maneira abrupta e o alto grau de faturamento não caracteriza atividades tectônicas atuais. Também justifica os fenômenos de alagamentos que ocorrem nessa região por se tratar de uma zona de alta diferenciação topográfica (observando o perfil W-E do mapa topográfico).

Outra zona termoestrutural caracterizada no estudo refere-se a região sudeste da bacia, que é marcada por suas fontes estruturais com profundidade variando de 4-5 $\mathrm{km}$ e apresentando valores de fluxo geotérmico anômalos com valores acima de $80 \mathrm{~mW} / \mathrm{m}^{2}$. Nesta zona verifica-se uma tendência dos lineamentos magnéticos de NE-SW para E-W. Por se tratar de uma borda de bacia sedimentar, a litologia apresenta baixos valores de condutividade térmica para essa região.

\section{Agradecimentos}

Este estudo é parte do projeto de iniciação cientifica da primeira autora no ON - LabGeot, projeto PIBIC.

\section{Referências}

Bhattacharyya, B.K., Leu, L.K., 1975. "Analysis of magnetic anomalies over Yellowstone National Park: mapping of Curie-point isothermal surface for geothermal reconnaissance" J. Geophysics. Res., 80: 4461-4465.

Bhattacharyya, B.K., Leu, L.K.1977. "Spectral analysis of gravity and magnetic anomalies due to rectangular prismatic bodies" Geophysics, 42:41-50.

CPRM, Serviço Geológico do Brasil. 2014. Data base online. Programa Geologia do Brasil. Rio de Janeiro.

Blakely, R.J., 1995. Potential Theory in Gravity \& Magnetic Applications. Cambridge University Press.

Brenner, T.L.; Ferrari, A.L. e Penha, H.M. 1980. Lineamentos estruturais do Nordeste do Estado do Rio de Janeiro. In: Congresso Brasileiro de Geologia, 32, 1980, Camboriú. Anais do Evento. SBG. 5:2551-2563.

Clark D.A. 1997. Magnetic petrophysics and magnetic petrology: aids to geological interpretation of magnetic surveys. AGSO J. Austr. Geol. Geophysics, 17:83-103
Gomes, A.J.L. 2003. Avaliação de Recursos Geotermais do Estado do Rio de Janeiro. Tese de Mestrado. ONMCTI.199p.

Gunn, P.J., Dentith, M.C. 1975. Magnetic responses associated with mineral deposits. AGSO Journal 17(2):145-158.

Nettleton L.L, Cannon J.R. 1962. Investigation of upward continuation system. Geophysics, 27: 796-806.

Silva, T.M. 2002. A estruturação geomorfologia do Planalto Atlântico no Estado do Rio de Janeiro. Rio de Janeiro, 265p. (Tese de Doutorado, Depto. Geografia IGEO/UFRJ).

Silva, L. C.; Cunha, H. C. S. 2000. Estudo geoambiental do estado do Rio de Janeiro. Brasília: CPRM-DEGET, 88 p.

Ross, J.L.S. 1985. Relevo brasileiro: uma nova proposta de classificação. Rev. Dept. Geog. FFLCH-USP, São Paulo. 4 : 25-39.

Thompson, D.T. 1982. EULDPH: A new technique for making computerassisted depth estimates from magnetic data.Geophysics, 47:31-37.

Valeriano, M. M. 2008. Topodata: guia para utilização de dados geomorfológicos locais. São José dos Campos, SP: INPE: Coordenação de Ensino, Documentação e Programas Especiais (INPE-15318-RPE/818). 72p. 